Cahiers de recherches médiévales

Figures mythiques médiévales auX $\mathrm{XIX}^{\mathrm{e}}$ et $\mathrm{XX}$ siècles

\title{
Roman et lyrisme courtois
}

Partonopeus de Blois et Galeran de Bretagne

Catherine Gaullier-Bougassas

\section{CpenEdition}

Journals

Édition électronique

URL : https://journals.openedition.org/crm/1853

DOI : $10.4000 / \mathrm{crm} .1853$

ISSN : 1955-2424

Éditeur

Honoré Champion

Édition imprimée

Date de publication : 15 décembre 2004

Pagination : 197-212

ISSN : $1272-9752$

Référence électronique

Catherine Gaullier-Bougassas, "Roman et lyrisme courtois », Cahiers de recherches médiévales [En ligne], 11 | 2004, mis en ligne le 10 octobre 2007, consulté le 15 décembre 2022. URL : http:// journals.openedition.org/crm/1853; DOI : https://doi.org/10.4000/crm.1853

Ce document a été généré automatiquement le 15 décembre 2022.

Tous droits réservés 


\title{
Roman et lyrisme courtois
}

\author{
Partonopeus de Blois et Galeran de Bretagne
}

\section{Catherine Gaullier-Bougassas}

1 Les liens du roman avec le lyrisme courtois des troubadours et des trouvères sont très anciens. Ils remontent aux fondements du genre romanesque tel qu'il s'élabore à partir de 1150 . Si le roman se démarque d'emblée de la chanson de geste par sa facture et son refus d'une thématique exclusivement guerrière, c'est en privilégiant un discours amoureux et en s'inspirant de la poésie lyrique courtoise et de sa célébration de la fin'amor. Vers 1150, un des tout premiers romans en langue française, le Conte de Floire et Blancheflor, met ainsi en scène un jeune héros espagnol, Floire, qui n'est pas un chevalier et qui, sans jamais combattre, se consacre entièrement à son amour pour Blancheflor ${ }^{1}$. Une éducation savante permet au couple de lire des livres d'amour «païens» et de se servir de la langue latine pour communiquer. Ils deviennent alors tous deux poètes:

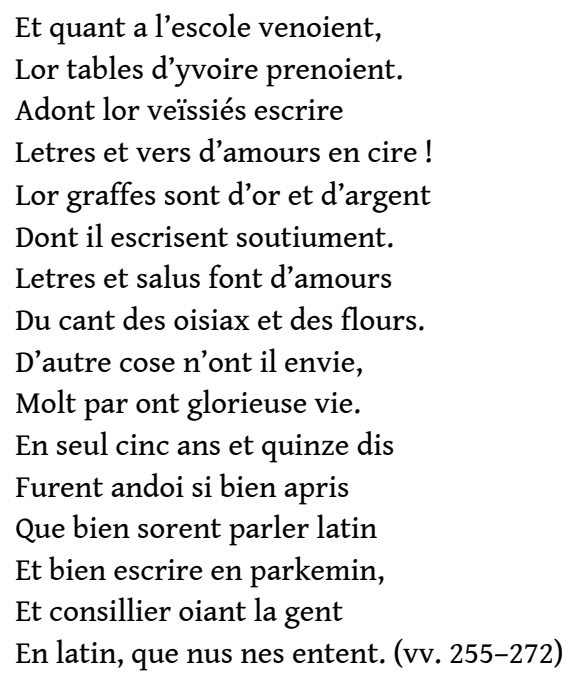

2 Le désir amoureux est déjà à l'origine de leur inspiration poétique, mais leurs vers et saluts d'amour ne sont pas insérés dans le cours du récit. Plus loin, la thématique courtoise du grand chant courtois est transposée dans les monologues de désespoir que 
prononce l'amant lorsqu'il est séparé de son amie. Quand Floire exhale sa souffrance, la vision courtoise de l'amour se mêle à la rhétorique de la plainte funèbre, du planctus, et son monologue, organisé en quatrains, retrouve une forme strophique (vv. 713-784).

Le procédé des insertions lyriques - l'enchâssement dans la narration de fragments de grands chants courtois - n'est toutefois attesté qu'à partir du XIII ${ }^{e}$ siècle, à l'initiative, semble-t-il, de Jean Renart ${ }^{2}$. Un jeu entre deux factures littéraires, deux dictions, se fait alors jour dans une même œuvre littéraire, avec tous les effets de sens que l'auteur peut lui donner. Dans le Guillaume de Dole, comme la critique l'a bien montré, Jean Renart construit un réseau de correspondances, d'analogies, entre le contenu des chansons qu'il reprend aux trouvères et l'intrigue amoureuse et/ou le portrait des personnages. Pour Conrad, les insertions lyriques tendent ainsi à se substituer aux analyses psychologiques et à montrer l'initiation progressive du personnage à la fin'amor.

Si ce procédé est encore absent des romans du XII siècle, les premiers romanciers s'inspirent pourtant largement de la poésie lyrique des troubadours. L'influence de la poésie courtoise s'inscrit avant tout sous la forme de la mise en récit de ses situationsclés, de la «narrativisation» des motifs principaux de la fin'amor: la mort d'amour, la folie d'amour, la prison d'amour ${ }^{3}$... Plusieurs romans du XII ${ }^{e}$ siècle ou du début du XIII ${ }^{e}$ siècle, antérieurs au Guillaume de Dole, représentent aussi des héros poètes et musiciens. Tristan et Yseut en sont bien sûr les plus célèbres. Si la musique joue un grand rôle dans leur destinée ${ }^{4}$, aucun poème n'est pourtant enchâssé dans la trame narrative des romans du XII ${ }^{\mathrm{e}}$ siècle.

Dans les années 1170, un roman anglo-normand moins connu, le Roman de Horn, écrit par un autre Thomas que l'auteur du Tristan, célèbre un héros, Horn, modèle de guerrier et de défenseur de la chrétienté contre les Sarrasins et aussi amant courtois exemplaire, de surcroît musicien virtuose ${ }^{5}$. L'auteur s'arrête longuement sur la scène qui le représente jouant à la harpe un lai et chantant ses amours pour une jeune princesse bretonne Rigmel. La poésie est la création non pas de Horn, mais du frère de la jeune fille. Grâce à l'amour qui développe ses talents musicaux, Horn en est l'interprète le plus virtuose et le plus passionné. Au-delà de la nouvelle preuve qu'elle donne de l'exemplarité amoureuse du jeune homme, la séquence a des implications narratives, puisqu'elle révèle l'identité de Horn, alors exilé, et qu'elle modifie le cours des événements en précipitant les retrouvailles des amants (laisses 134-137). L'auteur insiste aussi sur la grande diffusion dont le lai aurait bénéficié du vivant du couple et qui les aurait très vite transformés en amants mythiques. Aucune citation ne nous permet néanmoins de découvrir ce poème, alors qu'il est présenté comme l'une des matrices, fictive, du roman.

Une nouvelle évocation d'un poème courtois chanté, qui serait aux origines de l'écriture d'un roman, se retrouve dans une œuvre bien différente et un peu plus tardive, Galeran de Bretagne, qui date du tout début du XIII ${ }^{\mathrm{e}}$ siècle. Le romancier s'est avant tout inspiré du Lai de Frêne de Marie de France, pour composer son roman comme une amplification du récit bref de sa devancière ${ }^{6}$. Mais alors qu'il ne mentionne jamais l'existence du lai narratif de Marie de France, il choisit d'inventer un autre lai, lyrique, dont il attribue la composition à son héros Galeran. Sur le modèle de ce qu'affirment les troubadours et les trouvères, la naissance du désir amoureux métamorphose Galeran en un poète :

Or vont menant feste et revel.

Galeren a un son nouvel 
De l'amour qui le point trouvé,

Ou il a son cuer esprouvé :

Si a doulx chanz et plesans diz

Sans villanie et sans mesdiz,

Ci a plaie de doulceur pleine,

Qui les amans a pleurs rameine,

Quant ilz cuident jouer et rire.

Cil qui ce lay seulent escripre

L'apelent, au dit et au ton,

Le lay Galeren le Breton. (vv. 1971-1982)

Invoquer le succès dont le lai aurait joui, les multiples transcriptions dont il aurait fait l'objet contribue à authentifier son existence.

Enfin et surtout, l'auteur rapporte plus loin les circonstances de son interprétation avec un accompagnement à la harpe. Contrairement au Roman de Horn, la scène est intime, elle se déroule entre les deux amants, lors de leur premier rendez-vous amoureux, dans le verger du couvent. Deux traditions lyriques se réunissent alors pour magnifier la joie d'amour. Après une description de Frêne, de ses préparatifs et de ses parures, qui rappelle le lyrisme de mai et les reines de mai, les chansons à danser et notamment le cycle de «la bele Aelis» (vv.1983-2114), ce sont la plainte amoureuse que Galeran adresse à Frêne et la réponse de la jeune fille, puis la double interprétation du lai qui évoquent le grand chant courtois (vv. 2115-2327).

9 La progression de l'échange amoureux et des liens que chaque voix entretient avec la lyrique courtoise est alors signifiante. Tel un trouvère, Galeran prononce tout d'abord un hymne à l'amour qui trahit son adhésion à l'idéal littéraire de la fin'amor : il rend hommage à Amour, se soumet à toutes ses exigences, accepte sans condition les souffrances qu'il lui inflige. Le discours qu'il adresse à Frêne se caractérise par l'abstraction, sur le modèle du grand chant courtois. Quelques familiarités de langage donnent une couleur particulière à sa voix, mais, comme il les regrette et les corrige aussitôt, elles signalent qu'il continue de progresser dans son initiation à l'amour courtois. Seule l'inscription du prénom Frêne rappelle ce que le discours, en lui-même, tend à effacer: l'exploitation de la rhétorique courtoise dans une situation d'énonciation particulière, la mise en dialogue du chant courtois. En effet, dans son envolée lyrique, qui précède et annonce l'interprétation du lai, Galeran oublie toutes les conditions particulières de l'intrigue romanesque, les circonstances concrètes de sa liaison avec Frêne. Il s'adresse à elle comme si elle était la grande dame courtoise des poésies, alors que les amants sont confrontés à un problème de mésalliance, car Frêne, abandonnée par sa mère, est ravalée au statut d'enfant trouvée. Bien sûr, il cherche ainsi à la magnifier, mais en même temps son manque de prise sur la réalité annonce qu'il n'imposera pas à la société, par sa propre volonté, son union avec Frêne.

La transposition du chant courtois dans un dialogue romanesque se poursuit avec la réponse de la jeune femme. Or, Frêne ne consent pas d'emblée au portrait idéalisé que Galeran lui tend d'elle-même. Son attention se porte au contraire sur l'inadéquation de l'idéal courtois avec la situation concrète qu'elle vit. Elle commence même par remettre en cause - momentanément - la sincérité de Galeran. Une fois ses doutes écartés, elle continue de contester le discours courtois en insistant sur les difficultés réelles auxquelles elle est confrontée et qui sont étrangères à la vision courtoise de l'amour :

- Galeren, frere, doulx amis,

Ce dit Fresne, vous avez mis 
En moy amer tout vo pouoir :

Ce puis bien par dehors veoir ;

Mais je ne sçay jugier dedens :

Quant la parole est hors des dens,

Pence le cuer souvent tel chose

Qui est a la bouche forclose.

Ne dy mie pour vous mescroire :

Se mal souffrir fait amant croire

Qu'il soit loyaulx sans trahison,

Estre en devez un par rayson;

A loyal vous tieng sans mentir.

Non pourquant a douleur sentir

Vers moy ne vous aatissez,

Mais de l'amour tout me laissiez

Le fes et la cure et l'entente.

A tous biens recevoir suis tente,

Quant a m'amour un petit pens ;

Qu'il m'est avis en mon pourpens

Que j'ay aree pierre et greve,

Ou point n'a d'umour ne de seve.

Et comment me puet ferme faire

Amours qui m'est tout a contrere,

Qui me fait entendant la briche? (vv. 2165-2189)

11 Autrement dit, la parole féminine souligne la différence qui existe entre l'univers du roman et l'idéal littéraire abstrait, ritualisé, de la poésie courtoise. Sa contestation de la douleur de l'autre s'accompagne d'une peinture non courtoise de l'amour qui la déchire et qui, pense-t-elle, va la détruire.

12 L'aveu de l'amour - il s'agit ici de la première vraie rencontre amoureuse et de la première parole amoureuse - se présente donc d'abord comme une confrontation de deux plaintes et de deux discours sur l'amour. Dans les derniers vers qu'elle prononce, Frêne se départ néanmoins de son amertume et de sa peur pour consentir à ses sentiments et rejoindre son amant, elle finit par accepter le chant courtois qu'il lui adresse, par-delà les contraintes de la réalité.

13 C'est alors que prend place la scène d'interprétation du lai, qui correspond à l'apogée de la joie d'amour des amants. Transformé en trouvère par la passion amoureuse, l'amant présente son chant en hommage à sa dame. Lui offrant à son tour une image idéale de la reconnaissance de ses talents poétiques et de l'impact émotif de ses vers, elle trouve aussitôt l'accompagnement à la harpe le plus harmonieux, puis s'approprie les paroles de son amant:

«... commenciez, je herperay

Et en ma harpe l'aprenray. "

Il commence, celle l'escoute,

Qu'en la harpe ses doiz i boute.

Quant les notes a entendues

Au pletron les a estendues

Et atrempees a droit point.

Ce lay destraint Fresne et point.

Car cil qui si doulcement chante

Au commencier d'Amours se vante.

Aprés la blasme, aprés la prise ;

Plaine est de joye la reprise ;

D'Amours y est tout le contrere : 
Si est cruel, cy debonnaire,

Cil fait plourer et cil fait rire ;

En cestuy vers l'amant empire,

En cestuy le fait amender ;

En cest aultre l'estuet garder,

Et par de cza n'a point de soign ;

De ça pert tout a grant besoign,

Et de la rest tous esbaudiz.

Doulx est li chans et doulx li diz,

Et cil li chante tant et note

Qu'elle scet le dit et la note ;

A sa harpe l'a accordee

Qui estoit d'argent encordee. (vv. 2295-2320)

14 L'interprétation du lai crée un duo. La musique et la poésie réunissent les amants. Pour introduire le poème dans le récit, le narrateur omniscient préfère néanmoins à la citation et à la délégation de parole un résumé, qu'il prend en charge lui-même et développe assez longuement, en insistant sur le registre de la fin'amor.

La dame n'a pas encore accès à la création littéraire. Le narrateur la cantonne dans le rôle de harpiste accompagnatrice. C'est seulement beaucoup plus loin, à la fin du roman, lorsqu'elle se présente sous les traits d'une ménestrelle le jour des noces de Galeran avec sa sœur jumelle, qu'une inspiration poétique lui est soudainement accordée. L'auteur cite les deux premiers vers qu'elle compose sous l'emprise d'une émotion intense et dont le contenu peut rappeler les chansons de toile :

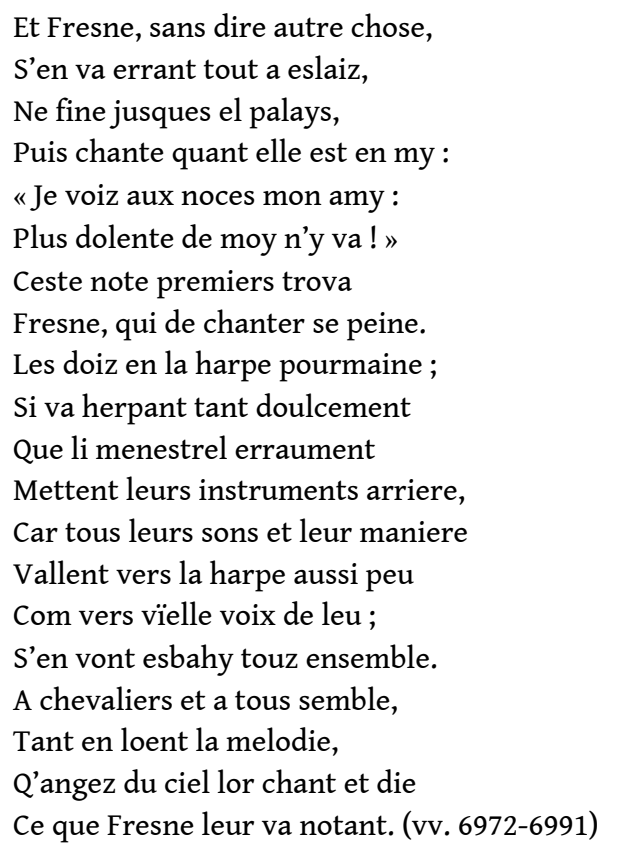

16 Puis, la reprise par la voix féminine du lai de Galeran, connu des seuls amants, provoque la reconnaissance :

Et Galeren se va matant Qui encore peu s'aparçoit ; Fresne l'esgarde, sil deçoit Et davant li de gré se porte. Par un doulx lay le desconforte ;

Les autres laiz, celuy a pris

Que Galeren li a apris. 
El dit ne mesprent n'en la note :

De Galeren le Breton note.

Si l'escoutent toutes et tuit ;

Des moz n'entent nulz le deduit

Fors que dui ; mais li chans est doulx,

Si les fait entendre a li tous.

Que que Galeren ot le lay,

Li sancs li mue sans delay,

Ne soit ou il est ne qu'il face ;

La couleur li voit en la face

Fresne muer, et sel voit taire. (vv. 6992-7009)

Sans jamais donner lieu à la moindre insertion lyrique, le chant courtois de Galeran, source imaginaire de la première partie du roman, avant la séparation des amants et leur parcours d'épreuves parallèles, remplit donc des fonctions narratives et symboliques essentielles. L'auteur est aussi l'un des premiers à célébrer avec originalité une femme poète et compositeur, une femme qui utilise ses dons d'artiste pour changer le cours de sa destinée et trouver le bonheur. Si Galeran puis Frêne, par leur création poétique et musicale, peuvent s'interpréter comme des figures de l'écrivain, ils n'apparaissent pas comme des doubles de l'auteur du roman, car ce dernier ne se donne pas les traits d'un amant courtois et d'un trouvère.

Les données sont différentes dans un autre roman, Partonopeus de Blois, qui est daté des années 1180 et qui, sans doute avant la fin du XII ${ }^{e}$ siècle, a été prolongé par une continuation $^{7}$. Cette œuvre, qui a joui d'un large succès, est un roman syncrétique, nourri des influences du lyrisme, de la chronique et de la chanson de geste. Son héros est un jeune homme de la famille des comtes de Blois, il est supposé vivre sous le règne $\mathrm{du}$ fils de Clovis, c'est-à-dire à une époque qui, pour les auditeurs de romans bretons, devait coïncider avec le règne d'Arthur. Toute la première partie se construit d'ailleurs à partir de la structure d'un conte morganien, comme si elle était l'amplification d'un lai breton, en même temps qu'une adaptation du conte de Cupidon et Psyché 8 .

Le roman entretient avec le lyrisme des trouvères des liens multiples et souvent novateurs dans les années 1180. L'auteur intègre en effet sous des formes multiples le discours lyrique au récit romanesque. Le phénomène de transposition dans le registre narratif de situations clés et de métaphores de la lyrique courtoise, que j'ai évoqué plus haut, est présent dans Partonopeus de Blois comme dans tant d'autres romans de l'époque, surtout après la transgression de l'interdit imposé par Mélior, lorsque la séparation des amants permet l'introduction de la thématique de l'amour de loin, du martyre et de la folie d'amour. La très longue séquence du tournoi montre ensuite comment, à l'image de ce qu'attestent par ailleurs les romans de Chrétien de Troyes, les liens entre l'amour et la chevalerie se substituent à ceux que les trouvères nouent entre l'amour et la création poétique. Si Partonopeus ne devient jamais un poète et ne s'illustre "que» par les armes, le narrateur dresse clairement un parallèle entre chevalier et clerc. Grâce à l'amour, le chevalier progresse en chevalerie autant que le clerc en clergie:

Ensi set amors engignier

Cascun home de son mestier :

Chevalier de chevalerie,

Et clerc d'amender sa clergie ;

Vilonie tolt et pereche,

Cortoisie done et noblece. 
Dame qui n'aime tieng a fole,

Poi pris son fait et sa parole.

Mais s'ele est bele, c'est a droit

C'on le requiere et ele otroit.

La moie amie enfin m'ocit,

Tant se deffent et escondist. (vv. 3525-3436)

En effet, l'une des originalités du roman et de sa continuation ${ }^{9}$ réside dans le nombre élevé et la richesse des interventions du narrateur-auteur ${ }^{10}$. Ce dernier s'impose avec insistance comme un amant courtois qui cherche désespérément à séduire sa dame mais se heurte toujours à sa dureté et à ses refus. Il ne cesse d'établir des liens entre ses personnages et lui, de comparer leurs sentiments aux siens, si bien qu'un discours pseudo-autobiographique se mêle au tissu narratif du roman. L'image de victime que le je amoureux de l'auteur s'attribue, sa célébration d'un amour courtois et la rhétorique qu'il emploie l'assimilent aussitôt à une figure de trouvère, même s'il ne prétend jamais avoir composé de chansons. Tel un trouvère, il répète inlassablement qu'il n'écrit que pour sa dame, que son inspiration prend son origine dans l'amour qu'il éprouve pour elle et qu'il lui offre son œuvre dans l'espoir de briser sa résistance.

Il consacre ainsi plus de quatre-vingts vers du prologue de son roman à une amplification du motif de la reverdie et à une célébration de son désir d'écriture par envoiseüre (v. 69), par joie d'amour, dans l'espoir de communiquer sa passion. Le roman se clôt aussi sur une adresse à sa dame. L'interruption de l'écriture est justifiée par l'absence de signes d'encouragement de cette dernière, bien que la possibilité d'une continuation s'offre encore, si elle le souhaite :

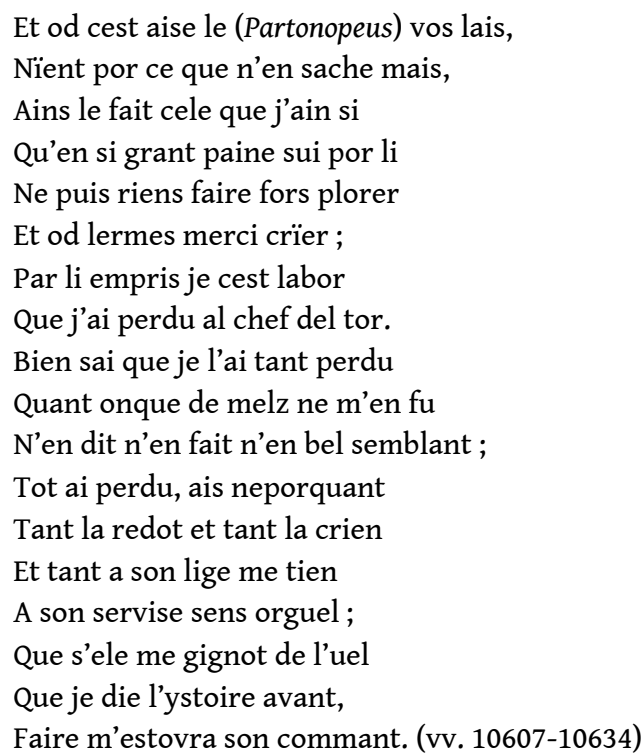

L'existence d'une continuation accrédite l'idée, une fiction sans doute, que la dame serait intervenue en sa faveur.

$\mathrm{Au}$ cœur de la continuation, le passage des couplets d'octosyllabes aux laisses épiques d'alexandrins est en outre expliqué comme une volonté de sa dame :

Je qui ceste chançon vos chant

Voeil que la fin voit amendant;

Jusques ci ai traitié la lime

Que chascuns couplés a sa rime.

Or le vous trairons par lons vers,

Si vous deviserons par mers. 
L'oevre en est cousteuse et plus fort,

Mais n'est ma vie ne ma mort

Que cele face son vouloir

De qui je ai petit espoir,

Car trop me donne d'autre part?

Mors sui se ainssi l'aimme endart. (vv. 1463-1474)

Il s'agit de l'une de ses interventions les plus mélodramatiques, où il annonce sa mort d'amour, en se comparant à une héroïne de l'Antiquité latine, Clytie, victime de Phoebus (vv.1475-1494). Enfin, l'épilogue de la continuation annonce une œuvre autobiographique, sans que l'auteur précise s'il pense à un récit ou à des chansons. Tel un trouvère, il garde le secret sur l'identité de la dame et la désigne par un surnom, Passe-Rose :

Partenopex maine grant joie ;

Avec lui Melior la bloie :

Mainent bon tans et boine vie.

Ainssi voussisse user ma vie

A servir la bele plaisant

Que je de fin cuer aime tant;

Et tant est bele a mon avis,

Rose de mai ne flor de lis

Ne si pueent a par tenir

Ne a sa biauté parvenir.

Ele s'apele Passe Rose ;

Icele ai en mon cuer enclose

Et la bonté ne puis escrivre :

Faire en porroie un autre livre. (vv. 3917-3929 de la continuation)

Que ces bribes d'autobiographie renvoient à un référent ou soient plus vraisemblablement pure fiction importe peu. L'essentiel est le jeu qu'elles instaurent entre les deux formes d'écriture, romanesque et lyrique, et l'image de l'écriture qu'elles dessinent. La connaissance intime que l'auteur a de la poésie lyrique laisse supposer qu'il a été un trouvère. Tout se passe comme si la dame, en le repoussant, rejetait le grand chant courtois et qu'il se sentait contraint, pour la séduire, d'exploiter une autre forme d'écriture, narrative. Il lui tiendrait alors des discours indirects, par le biais à la fois du récit de fiction et des adresses à la première personne qu'il destine en apparence à tous les auditeurs du roman. Autrement dit, ses interventions suggèrent que la fiction romanesque est écrite en fonction de sa situation personnelle: elle se substitue au discours lyrique à la première personne que la dame ne veut pas entendre. Grâce à la mise en scène de personnages étrangers, elle induit un éloignement spatial et temporel qui semble davantage susceptible de la séduire.

L'auteur insiste ainsi - et c'est original à la fin du XII ${ }^{\mathrm{e}}$ siècle - sur les liens profonds qui existeraient entre l'écriture d'un roman et sa propre subjectivité. Une telle inscription, aux origines de l'écriture du roman, de sa vie d'individu particulier est une innovation dans les années 1180 , même si le je de son discours amoureux correspond au je abstrait de la poésie lyrique et que jamais la moindre anecdote ou confidence personnelle n'annonce ce qui sera une spécificité du genre du dit à partir du XIII ${ }^{e}$ siècle. L'auteur participe ainsi à l'élaboration du genre romanesque au sens moderne du terme: l'univers qu'il crée, bien qu'il ne renonce pas complètement à l'alibi ou à la prétention historique ${ }^{11}$, serait pris en charge par sa conscience individuelle, par sa subjectivité. Les interventions du narrateur révèlent très souvent une projection consciente, avouée, dans les différents personnages. L'intrigue romanesque, avec l'invention du bonheur de 
Partonopeus, aurait pour finalité de remplir une fonction de compensation des échecs amoureux qu'il s'attribue. Le récit des amours de Mélior, de Partonopeus et du sultan de Perse lui permettrait d'épancher ses propres sentiments et de tenter d'influer sur sa dame et sur sa propre destinée ${ }^{12}$.

Par le biais de la fiction romanesque, il engage donc son amie, trop cruelle, à suivre l'exemple de Mélior, il lui adresse explicitement un rappel à l'ordre et justifie sa propre révolte. Les ressources didactiques du genre romanesque lui permettent de proposer des modèles de comportement féminin. Le discours lyrique du trouvère débouche d'ailleurs souvent sur un discours moralisateur qui s'attache aux dames en général. La sacralisation de l'amour courtois s'accompagne d'une dénonciation explicite de la misogynie des mauvais clercs, mais aussi d'une mise en cause des exigences excessives des dames trop hautaines ou de la chasteté des dames trop pieuses. Parallèlement, il exploite un autre ressort romanesque, ignoré de la lyrique du fait de son absence d'organisation narrative : il cherche à refermer sur son amie le piège de l'identification à une héroïne idéale.

Après le prologue, dans la première partie du roman pseudo-féerique, les interventions du je lyrique du narrateur sont d'abord discrètes. Le romancier s'efface le temps que la fascination suscitée par la découverte d'un univers merveilleux puisse pleinement s'exercer sur les auditeurs. Il invente une image sublime des splendeurs orientales et dresse le portrait d'une amante orientale qui possède toutes les vertus : beauté, sagesse, pureté morale, connaissance savante, sensualité, et offre au chevalier qu'elle a choisi sa personne et son empire. L'entrée de l'Orient dans le roman lui permet donc de représenter un idéal féminin qui comble les fantasmes masculins ${ }^{13}$. La dame orientale se distingue des dames occidentales par sa générosité et son acquiescement au bonheur. Auprès d'elle, l'ordre du parcours amoureux s'inverse, puisque l'union charnelle, accordée dès la première rencontre, précède la vision de sa beauté. Puis elle s'occupe de l'éducation du jeune homme et prépare son ascension politique. L'auteur intervient alors pour la déclarer plus courtoise que sa propre amie et condamner les dames occidentales qui, par leur obsession de la chasteté, trahiraient les valeurs courtoises. La princesse orientale incarne ainsi à ses yeux la dame courtoise telle qu'elle devrait être, à la fois toute-puissante et consentante à son amour. Le personnage de Mélior semble construit, du moins dans un premier temps, par inversion du portrait que le narrateur-auteur donne de sa propre amie :

En la cambre est el lit entrés ;

O Melior ot son delit,

Asés i giue, asés i rit,

Et n'est mervelle s'il a joie.

Car tant li set conter la bloie

Et de deduit et de grans sens,

Et de fais de l'ancien tens

Que nus ne set tant bien entendre

Qui ne puisse de lui apprendre.

Douce et soëf a le parole ;

C'est une riens qui molt m'afole :

Co ai de m'amie et nient plus,

Par tant m'i tieng et pens et mus ;

Et Dameldex, qui ne menti,

Me doinst qu'el m'aint si com j'aim li,

Et qu'a moi pere se francise,

Car ce n'ert ja que autre eslise. 
Partonopeus a son delit.

Li parlers de lui molt m'ocit,

Car il a tos biens de s'amie,

Jo n'en ai riens qui ne m'ocie.

Il ne le voit, mais a loisir

Le sent et en fait son plaisir ;

Je voi la moie, et n'en faç rien,

J'en ai le mal, et il le bien. (vv. 1862-1886)

\section{condamnation de la chasteté exce
l'idéal courtois (vv. 8009-8068)}

Poi est a dames de richece,

Ne de grans dons ne de largece,

Ne bel parler ne cortoisie

Nes puet torner de caste vie.

Chascune seit ja son psaltier,

Si vait bien ainz jor al mostier ;

Illoc font lor afflictions

Od larmes et od orisons,

S'i demeurent duc'a la prime.

Tant traient ceste sainte lime

Que de Deu sunt illuminees

Et del Saint Espir garantees.

Por nïent les requiert om mais. (vv. 8019-8031) ${ }^{14}$

31 L'histoire de Mélior est là pour témoigner que le don de la dame à son amant n'est pas synonyme de déchéance et de dégradation, mais qu'au contraire il conduit les deux amants à progresser dans l'adhésion au code courtois.

La transgression de l'interdit par Partonopeus modifie ensuite les rapports de force dans le couple. Dans le même temps, les interventions du narrateur, de plus en plus nombreuses, manifestent bien davantage la présence de son je lyrique. Dépossédée subitement de ses pouvoirs d'enchanteresse, Mélior perd sa toute-puissance et doit se soumettre à l'autorité de ses barons. Elle se rapproche de la dame courtoise cruelle que chantent tant de trouvères, car elle choisit de punir Partonopeus en l'exilant, alors que sœur lui conseille de pardonner et lui indique un stratagème pour contraindre ses barons à accepter son mariage (vv. 4923-5060). Le roman s'attarde longuement sur le dialogue des deux sœurs, puis sur le désespoir et la folie de Partonopeus. Les relations que le narrateur établit entre ses deux personnages de roman, sa propre dame et luimême, se modifient. Le portrait de Mélior tend à coïncider avec celui de sa propre amie. Le scénario narratif que l'auteur reprend aux lais féeriques lui sert à instruire une nouvelle fois, autrement, le procès de sa dame et des dames courtoises trop hautaines. Cette condamnation de Mélior est néanmoins de courte durée. En effet, la princesse se repent et expie sa trop grande sévérité par un martyre d'amour : elle exhale de longues plaintes pour regretter son orgueil et exprimer le désir de mort qui la submerge si elle est séparée à tout jamais de son amant. Le narrateur s'identifie alors à la jeune femme qui souffre et oppose la loyauté de son amour à l'indifférence de sa propre dame (vv. 8603-8616, 9015-9038, 10101-10124). 

précis entre sa situation personnelle et celle de ses personnages, imposent donc l'idée que la fiction romanesque est inventée, pensée, comme une démonstration et un instrument au service des intérêts du je amoureux et lyrique du narrateur-auteur. Selon la fiction autobiographique, la dame elle-même semble refuser l'hommage du grand chant courtois et préférer l'univers du roman. L'auteur exploiterait alors les ressources du genre romanesque - le ressort de l'identification de l'auteur et de l'auditeur aux personnages, les ambitions didactiques - pour renouveler sa déclaration d'amour à sa dame et aussi lui lancer un avertissement implicite.

Plus loin, au cœur de la continuation, le goût pour les chansons de geste qui est prêté à la dame, au-delà d'une possible mise à l'épreuve des ressources du poète, semble manifester de sa part une rupture plus radicale avec la lyrique courtoise et la thématique amoureuse, soit un nouveau rejet des sentiments du poète. Tout se passe alors comme si l'auteur se jouait de la demande de sa dame, car cette fin attendue d'un discours sur l'amour n'a pas lieu. Bien au contraire, l'adoption de la facture des chansons de geste coïncide avec le moment où une nouvelle figure d'amant courtois exemplaire, le sultan de Perse, accède au rôle de héros principal. Célébré comme amant courtois exemplaire, il devient poète et écrit pour sa dame deux poèmes d'amour qui sont insérés dans le récit.

La continuation offre ainsi deux exemples d'insertions poétiques sans doute antérieurs au Guillaume de Dole et différents de ceux de Jean Renart. Les citations sont des poèmes inventés par l'auteur, ils n'existent pas antérieurement et n'entretiennent aucun lien avec la musique et le chant. Le premier est un quatrain et le second, qui correspond à un genre poétique pratiqué par les troubadours et les trouvères, est un long salut d'amour de 123 vers. Le sultan n'est pas musicien, il envoie ses poésies sous forme de messages écrits, de lettres, que la dame devra lire. L'auteur décrit alors précisément les attitudes d'un poète amoureux en train de composer, les sentiments contradictoires qui l'animent, ses hésitations, comment il rature, déchire, réécrit un poème sans cesse recommencé :

Il est clers bons, eslis, et des ars enseigniés ;

Par brief li volt mander com il est traveilliés

Et com son joie est lonc de son cuer essilliés,

Que ja ne l'avra mais se ne l'en prent pitiés.

Il s'asiet en son lit, se si est aesiés :

A un grant oreillier de paile est apuiés,

Si s'atorne a escrire, si s'est appareilliés.

Si est li briés molt tost et bel encommenciés,

Et puis autresi tost il i rest depeciés.

Dont est fait, dont deffait, dont empris, dont lessiés,

Dont monte ses desdains, et puis est tost plaissiés.

Dont dist qu'il est frarins, dont renorte se fiés,

Dont est ses cuers par tout, dont est il empiriés.

Dont a si bon espoir qu'il en est outre liés,

Et dont si grant paour qu'il en est tous chargiés.

Dont se detort trestous com uns serpens bleciés,

Et dont se gist en pais com une escorce viés.

Ses cuers est departis, si est si detrenchiés,

Ne se tenist pas tant que il se fust seigniés.

Ore se rest par soi couvers et despoilliés :

Trestous ses chamberlens en a il enchaciés ; 
Seul se despoille et vest. En seant redreciés

Un brief fait et escrit qui molt est envoisiés :

De mos et de samblans est il entrelaciés. (vv. 3024-3047)

L'une des originalités de la séquence est de regrouper différentes formes littéraires : les laisses épiques d'alexandrins, les décasyllabes avec rimes équivoquées du salut d'amour, le quatrain en octosyllabes, puis le retour aux octosyllabes à rimes plates du roman, à la fin du salut d'amour. Procédé exceptionnel dans le roman en langue d'oïl, le mélange des mètres devait provoquer la surprise des auditeurs :

Vilains n'en gouste pas, car c'est trestous daintiés,

Ains s'en esbahist tant quant il voit ses daintiés ;

Petis est et soutis. Or öés et taisiés :

«Par Melior en ma mort vif

Qui me lie ore en fort estrif ;

La vie est nove c'ai volu

Qui vie et joie m'a tolu.»

Quant les mos ot escris, s'a les dis regardés ;

Li mot son engignous et li briés bien formés,

Mais ses cuers engignous est si en grans lastés

Trop li samble petit a l'aviser ses ses.

Greignour brief i estuet; de ce s'est porpenssés.

Bien (en)fait un molt tost qui est greignor d'assés,

Et nous le vous dirons s'entendre le volés.

Onques devant cestui ne fu tel manouvrés :

Par un mot en deus sens est li couplés finés,

Si est trestous li briés jusqu'a la fin menés.

[...]

"Salus vos mande vos premiers drus a joie;

Nel puis oïr sans riens por duel que j'aie.

Salut vous mant a certes que je port ;

En haute mer m'avés geté du port ». (v. 3048-3074)

Après le récit du mariage de Partonopeus, le narrateur-auteur de la continuation, qui se présente aussi comme l'auteur du roman et insiste sur son échec amoureux, choisit désormais de s'identifier au sultan de Perse, c'est-à-dire à un personnage a priori très éloigné de lui-même: un Oriental musulman, qui ne s'est pas converti, qui déclare la guerre à Partonopeus et menace dangereusement l'Empire byzantin. Le roman rompt alors définitivement avec les stéréotypes épiques en magnifiant le sultan comme une figure idéale d'amant chevalier et poète. Le prince perse exprime sa souffrance, son martyre d'amour, il dénonce la cruauté de Mélior et tient un discours contre la chasteté de la dame qui coïncide exactement avec celui du narrateur dans le roman :

... ele tient une autre foi,

Qu'ele a une novele loi

D'un Diu qui aimme chaasté,

Qui onques en tout son aé

N'ama d'amors, tant par est fiers. (vv. 3269-3273, voir aussi 3273-3290)

Double de l'auteur, il lui offre une nouvelle voix pour exprimer sa désillusion amoureuse et prouver son attachement à la poésie courtoise que refuse sa dame. Le long salut d'amour tend à apparaître comme l'aboutissement du roman et de sa continuation, comme le poème que l'auteur aurait voulu écrire pour sa dame et qu'il lui adresse indirectement. La parole poétique, bien que rejetée, refoulée, ressurgit, transformée en message écrit, en lettre, et composée par un étranger à la fois menaçant et proche par son adhésion à l'idéal courtois et ses talents d'écriture. Mais elle se 
heurte à un échec, qui semble redoubler celui de l'auteur : Mélior exprime à nouveau son désamour pour le sultan. La poésie amoureuse offerte en hommage ne soulève aucune émotion dans le cœur de la dame, on ignore même si Mélior accepte ou non de lire la lettre.

Le personnage du sultan, sur lequel le continuateur projette ses sentiments et son savoir-faire littéraire, se substitue ainsi à un autre double implicite de l'auteur que célébrait le roman. Il s'agit de Mélior elle-même, la conteuse passionnante et l'enchanteresse experte dans la mise en scène de spectacles. Elle appelle elle-même œuvres les scénarios qu'elle invente et les récits historiques qu'elle narre à Partonopeus exercent sur lui une profonde fascination (vv. 1866-1870). Son personnage semble alors offrir une image rêvée de l'auteur de roman, du romancier à succès, alors que le poète courtois qu'est le sultan reste prisonnier de sa solitude et de son échec.

Les liens profonds que le roman de Partonopeus entretient avec le lyrisme courtois signifient peut-être la reconnaissance par l'auteur d'un passage nécessaire, choisi mais pour une part regretté, de la poésie lyrique à la narration romanesque, du discours sur soi à la première personne au récit fictionnel sur autrui. Au-delà des bribes d'autobiographie ou de pseudo-autobiographie, il est aussi tentant d'interpréter l'amie de l'auteur - et non Mélior - comme un double de l'auditeur à conquérir, de l'auditeur que l'écrivain cherche à fasciner, à émerveiller. Or l'auteur de Partonopeus semble considérer que l'écriture du roman est la plus apte à susciter ce désir et ce plaisir de l'Autre. Une nostalgie pour le discours poétique des trouvères affleure néanmoins, non seulement parce qu'il est transposé dans l'univers du roman, mais aussi parce que la continuation se termine sur un portrait magnifié de poète courtois - le fascinant sultan -, et sur l'enchâssement de deux de ses poèmes.

\section{NOTES}

1.Éd. et trad. J.-L. Leclanche, Paris, Champion, 2003 (Classiques Champion).

2.Jean Renart, Le Roman de la Rose ou de Guillaume de Dole, éd. F. Lecoy, Paris, Champion, 1962. Voir M. Zink, Roman rose et rose rouge : le Roman de la Rose ou le Guillaume de Dole, Paris, Nizet, 1979 ; M.-R. Jung, « L'empereur Conrad chanteur de poésie lyrique. Fiction et vérité dans le Roman de la Rose de Jean Renart », Romania, t. 101, 1980, pp. 35-50 ; E. Baumgartner, «Les citations lyriques dans le Roman de la Rose de Jean Renart ", Romance Philology, t. 35, 1981, pp. 260-266 ; M. Accarie, « Les fonctions des chansons du Guillaume de Dole », Mélanges J. Larmat, 1983, pp. 13-29; D. Duport, « Les chansons dans le Guillaume de Dole ", Et c'est la fin pour quoy sommes ensemble, Hommage à Jean Dufournet, Paris, Champion, 1993, t. 2, pp. 513-524.

3.Ce phénomène, qui parcourt tout le roman du XII ${ }^{\mathrm{e}}$ siècle, et au-delà, a été bien étudié, notamment pour le Lancelot de Chrétien de Troyes (voir E. Baumgartner, Chrétien de Troyes, Yvain, Lancelot, la charrette et le lion, Paris, PUF, 1992, pp. 19-20, 59-85 (Études littéraires)). Les monologues de plainte amoureuse constituent toujours un espace 
privilégié pour l'inscription de la rhétorique des trouvères dans la continuité des octosyllabes romanesques.

4.Rappelons que dans les récits en vers du XII ${ }^{e}$ siècle, c'est surtout Tristan qui est doté de talents poétiques. Il serait ainsi le premier auteur du Lai du Chèvrefeuille et Marie de France retrace l'origine du poème et les conditions de son écriture par l'amant d'Iseut. Au sujet des talents de musicienne et peut-être de trobairitz d'Iseut, la scène la plus célèbre des œuvres du XII ${ }^{e}$ siècle est celle du roman de Thomas, lorsqu'il décrit la reine en train d'interpréter et peut-être de composer à la harpe le Lai de Guiron, sur la légende du cœur mangé (Thomas, Le Roman de Tristan, éd. F. Lecoy, trad. E. Baumgartner et I. Short, Paris, Champion, 2003, vv. 987-1000 (Classiques Champion) ; Marie de France, le Lai du Chèvrefeuille, éd. M. Demaules, Tristan et Yseut, les premières versions européennes, dir. Ch. Marchello-Nizia, Paris, Gallimard, 1995, pp. 213-216, 1287-1309. Voir D. Buschinger, «La musique dans le Tristan de Thomas et le Tristan de Gottfried, quelques jalons ", Musique, littérature et société au Moyen Âge, Amiens, 1980, pp. 171-185 ; E. Baumgartner, "Lyrisme et roman : du Lai de Guirun au Lai du Chèvrefeuille », De l'Histoire de Troie au Livre du Graal, Orléans, Paradigme, 1994, pp. 317-323 ; V. Bertolucci Pizzorusso, «L'Arpa d'Isotta : variazioni testuali e figurative », Mélanges Ph. Ménard, Paris, Champion, 1998, t. I, pp. 101-109).

5.Thomas, Le Roman de Horn, éd. M. K. Pope, Oxford, 1955 et 1964, 2 vol.

6.Galeran de Bretagne, éd. L. Foulet, Paris, Champion, 1975.

7.Partonopeus de Blois (et sa continuation ), éd. J. Gildea, Villanova, 1967-1968, 2 vol.

8.Rappelons que l'auteur imagine un empire byzantin tombé en quenouille. L'héritière, Mélior, s'éprend de Partonopeus et, telle une fée, organise sa venue dans une ville qui évoque Constantinople, avant de s'offrir à lui. Elle lui fixe un interdit : il doit vivre deux ans et demi dans son palais somptueux, protégé des autres par une bulle d'invisibilité. Elle le rejoindra chaque nuit, mais il ne devra jamais chercher à la voir. Il transgresse l'interdit et sa désobéissance entraîne deux grandes conséquences, l'une inattendue : elle perd ses pouvoirs magiques et révèle sa fausse nature de fée, l'autre attendue : elle le rejette et le plonge dans le désespoir. Une longue période de séparation s'ensuit, marquée par l'errance et la folie pour le héros. Partonopeus participe finalement à un tournoi que les barons byzantins organisent pour désigner l'époux de Mélior. Il le gagne après avoir affronté le sultan de Perse, son rival amoureux. Sur la structure du conte féerique, voir L. Harf-Lancner, Les Fées au Moyen Âge, Morgane et Mélusine ou la naissance des fées, Paris, Champion, 1984, rééd. 1991 ; sur le syncrétisme du roman, M. Tomaryn-Bruckner, Shaping Romance, Interpretation, Truth and Closure in TwelfthCentury French Fictions, Philadelphia, 1993, pp. 109-156.

9.Impossible de savoir si c'est le même auteur qui a composé le roman et la continuation. Quoi qu'il en soit, la continuation prolonge les fils que le roman laisse en suspens et en respecte l'esprit.

10.Elles ont déjà suscité des études : J. L. Grigsby, «The Narrator in Partonopeu de Blois, le Bel Inconnu, and Joufroi de Poitiers ", Romance Philology, 21, 1968, pp. 536-545 ; M. Zink, La subjectivité littéraire, Paris, PUF, 1985, pp. 44-46 ; R. L. Krueger, « The author's voice : narrators, audience and the problem of interpretation », The Legacy of Chrétien de Troyes, Amsterdam, Rodopi, 1987, pp. 125-129, « Textuality and Performance in Partonopeu de Blois ", Assays : Critical Approaches to Medieval and Renaissance Texts, éd. P. Knapp, Pittsburgh, 1985, III, pp. 57-72 ; L. Walters, « The poet-narrator's address to his lady as structural device in Partonopeu de Blois ", Medium Aevum, 61, 1992, pp. 229-241. 
11.La reprise au début du récit de la légende des origines troyennes des Mérovingiens vise à donner à l'œuvre le statut d'une chronique familiale en langue vernaculaire sur les origines du lignage des comtes de Blois.

12.Le procédé sera bien sûr imité au XIII ${ }^{\mathrm{e}}$ siècle, notamment par Renaut de Beaujeu dans Le Bel Inconnu.

13.Sur les images romanesques de l'Orient, voir notre étude La Tentation de l'Orient dans le roman médiéval, Sur l'imaginaire médiéval de l'Autre, Paris, Champion, 2003 (Nouvelle Bibliothèque du Moyen Âge).

14.Voir aussi sur ce thème les vers 6261-6294, 7095-7178.

\section{AUTEUR}

\section{CATHERINE GAULLIER-BOUGASSAS}

Université de Paris-III Sorbonne nouvelle 